\title{
Special Corner: representational content and cognitive abilities
}

\author{
Holger Lyre
}

Received: 25 November 2008/ Accepted: 28 November 2008/Published online: 19 December 2008

(C) Marta Olivetti Belardinelli and Springer-Verlag 2008

Questions about the nature of mental representations and cognitive content and the explanatory value of ascribing representational powers to cognitive systems belong to the most virulent foundational issues in the cognitive sciences. This Special Corner focuses on whether-and if so which-role representational content plays and how it helps to understand and to explain our cognitive abilities. This is of eminent interest both for a conceptual analysis of the modeling task in the cognitive sciences as well as for a deeper philosophical understanding of cognition. The three papers in this Corner try to address these issues from different angles, they result from an International Workshop under the title "Understanding Cognitive Abilities-Logical and Representational Aspects", which took place at the University of Bonn end of August 2007.

The concept of representation is extremely dazzling. On the one hand, it seems to be the most obvious theoretical term in the cognitive sciences, indispensable in modelling and explaining the cognitive machinery. On the other hand, the notion of representation adamantly resists any attempts to "naturalize" it or, at least, to develop a widely agreed and convincing theory of representational content. Representations carry content, they are, as philosophers put it, "about" something (mostly the object of an intentional attitude such as belief or knowledge). And it is this asymmetric aboutness-relation which resists an explanation in purely naturalistic terms. How can it be that a mental representation, a neural brain state, say, is "about" something? How can such a relation be integrated into a scientific picture?

H. Lyre $(\square)$

Philosophy Department, University of Bielefeld,

Bielefeld, Germany

e-mail: lyre@uni-bielefeld.de; lyre@uni-bonn.de
There do, of course, exist naturalistic accounts of representation in philosophy, first and foremost causal theories of meaning and teleosemantics. But such theories suffer from various conceptual problems-most prominently the problem of misrepresentation (i.e. the problem that naturalistic theories have troubles to account for misrepresentation, which is mainly due to the fact that the aboutness-relation is seemingly asymmetric). Because of these unresolved problems and at the same time triggered by the paradigmatic developments under the headings of dynamicism, embodiment and situated cognition, not a few cognitive scientists and more and more philosophers have argued that the notion of representation should be dismissed altogether, that we should rather model and reconstruct cognition in more direct, physical terms. But this seems to fall short of the overwhelming practice of modelling cognitive systems. The authors in this Special Corner, therefore, adopt a more conservative attitude and try to defend the notion of representation by making clear that in order to model cognitive systems and, especially, to understand cognitive abilities, the concept of representation is of no alternative. The Special Corner also puts a certain emphasis on non-propositional and non-conceptual content and its connection to "know how".

Let us consider the papers in more detail. In the first paper "The Role of Representation in Computation", Jon Opie and Gerard O'Brien try to defend the explanatory role representations play in both digital and analog computations. Despite much recent anti-representational scepticism from dynamicist and embodied cognition accounts, the authors not only try to defend the concept of representation but also analyse why, in their opinion, the role of representation has been misconceived by anti-representationalists. As Opie and O'Brien put it, representation plays an indirect role in digital, but a direct role in analog computation. While in the 
former case representations serve to shape the syntactic rules, in the latter the cognitive system itself shows structural resemblance to the target domain. Here, the authors link their presentation to a "Structuralist Theory of Mental Representation".

Emphasis on such a structuralist view plays also a role in the second paper "Functional Role Theories of Representation and Content Explanation" by Andreas Bartels and Mark May. In it the authors aim to show that, contrary to a widespread opinion, functional role theories of representation do not fail to account for content explanations of human and animal behavior. They argue that structural resemblance allows for a definition of functional roles by their specific contribution within the cognitive processing, enabling, for instance, to explain successful homing performances in ants based on path integration mechanisms.

In the third and last paper of this Special Corner, "How to Reason without Words: Inference as Categorization", Ronaldo Vigo and Colin Allen try to show that it is possible to account for an inference-based conception of rationality even at the non-linguistic level. The authors present a case study of perceptual categorization according to the abstract modal properties of stimuli. This allows them to reconstruct the perceptual capacities underlying formal reasoning as subsymbolic processes for similarity assessment, discrimination and categorization. If Vigo and Allen are right, this opens an understanding of reasoning as a cognitive ability not only for humans but even for languageless animals. 